\title{
Adesão ao seguimento nutricional ambulatorial pós-cirurgia bariátrica e fatores associados ${ }^{1}$
}

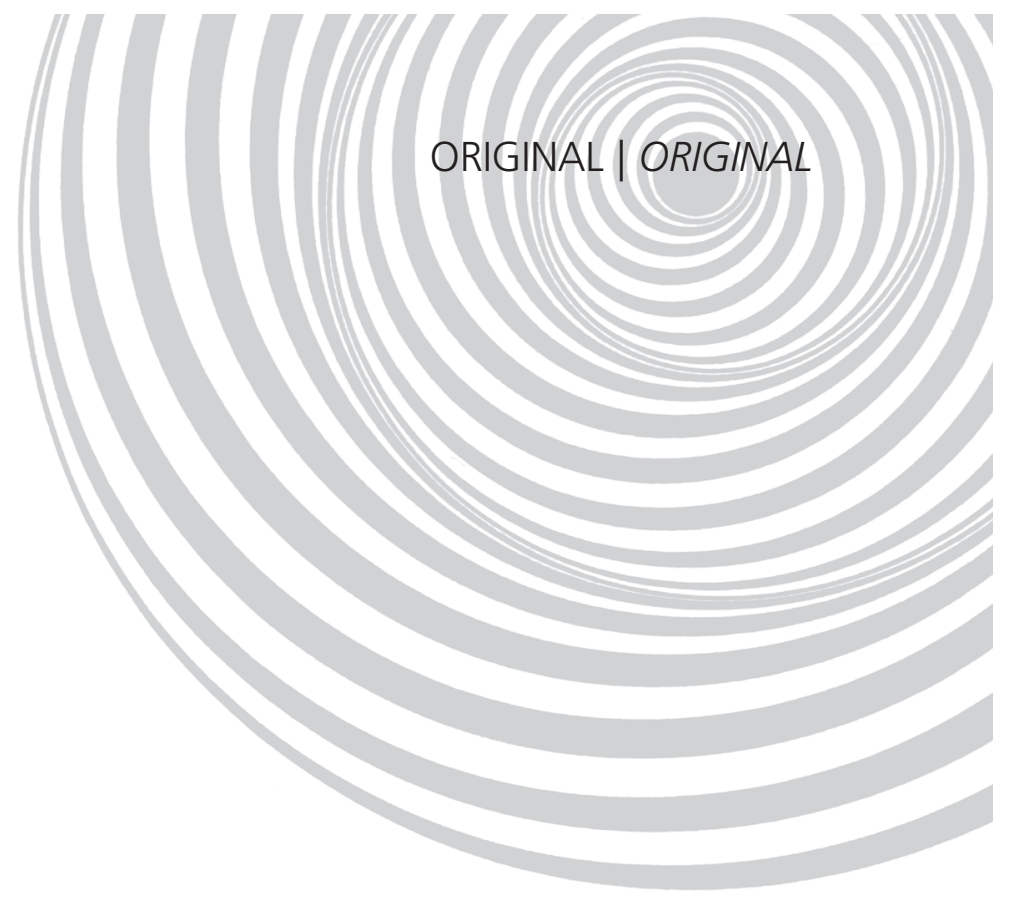

\author{
Adherence to outpatient nutritional \\ follow-up after bariatric surgery \\ and associated factors
}

Veruska Magalhães SCABIM²

Jose ELUF-NETO 3

Beatriz Helena TESS ${ }^{3}$

RE S U M O

\section{Objetivo}

Estimar a prevalência da adesão ao seguimento nutricional ambulatorial pós-cirúrgico e avaliar sua associação com fatores selecionados em indivíduos submetidos à cirurgia bariátrica.

\section{Métodos}

Estudo de coorte retrospectiva com base na revisão de dados pós-operatórios de 241 prontuários de adultos submetidos à gastroplastia redutora com derivação em Y de Roux entre 2006 e 2008. Considerou-se aderente o indivíduo que compareceu a quatro ou mais consultas nutricionais nos 12 primeiros meses após a cirurgia. Para investigar a associação entre adesão ao seguimento nutricional e idade, sexo, estado conjugal, escolaridade, situação empregatícia, distância entre a residência e o hospital, estratégias para perda de peso no período préoperatório, índice de massa corporal no pré-cirúrgico imediato, presença de comorbidades e duração da internação pós-operatória, foram calculadas razões de prevalência e utilizou-se regressão múltipla de Poisson.

\section{Resultados}

A prevalência de adesão foi de $56 \%$ (IC95\%=49,7-62,3) nessa população predominantemente feminina $(80,9 \%)$, com média de idade de 44,4 anos $(\mathrm{DP}=11,6)$ e de IMC pré-operatório de $47,2 \mathrm{~kg} / \mathrm{m}^{2}$ (DP=6,2). Dos

\footnotetext{
$\overline{1}$ Artigo elaborado a partir da dissertação de VM SCABIM, intitulada "Adesão ao seguimento nutricional ambulatorial pós-operatório de cirurgia bariátrica no Hospital das Clínicas da Faculdade de Medicina da Universidade de São Paulo - SP - Brasil". Universidade de São Paulo; 2012.

2 Universidade de São Paulo, Faculdade de Medicina, Hospital das Clínicas, Instituto Central, Divisão de Nutrição e Dietética. São Paulo, SP, Brasil.

${ }^{3}$ Universidade de São Paulo, Faculdade de Medicina, Departamento de Medicina Preventiva. Av. Dr. Arnaldo, 455, $2^{\circ}$ andar, Cerqueira César, 01246-903, São Paulo, SP, Brasil, Correspondência para/Correspondence to: BH TESS. E-mail: <beatriz.tess@usp.br>.
} 
498 | VM SCABIM et al.

fatores estudados, somente a duração da internação pós-operatória igual ou superior a 6 dias mostrou-se significativamente associada à adesão após análise ajustada por sexo e idade ( $R P=1,46$; IC95\%=1,15-1,86).

\section{Conclusão}

A prevalência de adesão encontrada foi semelhante às de estudos internacionais, mas baixa considerando-se $75 \%$ como referência. A maior adesão observada nos indivíduos com internação pós-operatória prolongada pode sugerir que o maior contato com a equipe multiprofissional aumente a percepção da necessidade de cuidados com a saúde em longo prazo.

Termos de indexação: Avaliação nutricional. Cirurgia bariátrica. Estudos observacionais. Obesidade. Período pós-operatório.

\section{A B S T R A C T}

\section{Objective}

This study estimated the prevalence of adherence to outpatient postoperative nutritional follow-up after bariatric surgery and analyzed the association between adherence and selected factors.

\section{Methods}

A total of 241 records of female and male adults who underwent Roux-en-Y gastric bypass between 2006 and 2008 were reviewed for this retrospective cohort study. The data included the preoperative and postoperative periods. Individuals were considered compliant when they attended four or more nutritional appointments in the first 12 months after surgery. Prevalence ratios were calculated for estimating the association between adherence to postoperative nutritional follow-up and factors such as age, gender, marital status, education level, employment status, distance between home and hospital, weight loss strategies used during the preoperative period, body mass index immediately before surgery, presence of comorbidities and duration of hospital stay after surgery, and the Poisson multiple regression was used for adjusted analysis.

\section{Results}

Adherence prevalence was $56.0 \%$ (C195\% 49.7-62.3). Women composed $80.9 \%$ of the population; the mean age of the sample was 44.4 years $(S D=11.6)$ and mean preoperative body mass index was $47.2 \mathrm{~kg} / \mathrm{m}^{2}(S D=6.2)$. Among the study factors, only duration of postoperative hospital stay was significantly associated with adherence after adjustment for age and gender ( $P R=1.46$ C/95\% 1.15-1.86).

\section{Conclusion}

The adherence prevalence of this population was similar to those of foreign studies, but below the minimum prevalence of $75 \%$ considered reference. The greater adherence of individuals with longer postoperative hospital stays may have stemmed from their prolonged interaction with the multidisciplinary team, which may have increased their awareness of disease severity and need of long-term health care.

Indexing terms: Nutrition assessment. Bariatric surgery. Observational studies. Obesity. Postoperative period.

\section{N T R O D U ÇÃ O}

A obesidade é uma doença crônica global de incidência crescente e que se tornou nas três últimas décadas um dos maiores problemas de saúde pública das sociedades urbanas'. A obesidade mórbida ou grau III, segundo classificação proposta pela Organização Mundial da Saúde², denota maior severidade da doença ${ }^{3}$. Com base em dados de inquéritos populacionais por amostragem realizados pela Sociedade Brasileira de Cirurgia Bariátrica e Metabólica (SBCBM), estima- -se uma prevalência de obesidade mórbida em adultos com 18 anos de idade ou mais de $3 \%$, sendo $2 \%$ entre os homens e $4 \%$ entre as mulheres $^{4}$.

Em 1999, o Ministério da Saúde reconheceu o tratamento cirúrgico das obesidades grau II, com comorbidades, e grau III segundo a classificação da Organização Mundial da Saúde (OMS) como uma alternativa eficaz para o controle dessa doença e o incluiu entre os procedimentos pagos pelo Sistema Único de Saúde (SUS) ${ }^{5}$. Entre 1999 
e 2006, foram realizadas mais de 10000 cirurgias bariátricas no Brasil pelo SUS ${ }^{6}$, sendo a gastroplastia com derivação em $Y$ de Roux, ou Derivação Gástrica em Y de Roux (DGYR), o tipo de cirurgia mais realizado. Por ser uma cirurgia de grande porte com risco de complicações a longo prazo, preconiza-se o acompanhamento contínuo dos indivíduos operados por equipe multidisciplinar? O seguimento nutricional quando realizado rotineiramente pode identificar e intervir precocemente em situações pós-operatórias de perda insuficiente ou reganho do excesso de peso e deficiências nutricionais e, dessa forma, contribuir para resultados favoráveis do tratamento cirúrgico da obesidade ${ }^{8,9}$.

A prevalência de adesão ao seguimento pós-operatório tem sido considerada um importante critério para avaliação dos serviços que realizam cirurgia bariátrica. Segundo a Surgical Review Corporation ${ }^{10}$, dentre os quesitos para a obtenção de certificado de centro de excelência, a instituição deve apresentar dados sobre o monitoramento da saúde de no mínimo $75 \%$ dos indivíduos por um período de 5 anos de seguimento após a cirurgia.

O comparecimento escasso ou irregular dos indivíduos às consultas agendadas no período pós-operatório tem sido observado em vários estudos em populações norte-americanas ${ }^{11-17}$, com taxas de adesão entre $40 \%$ e $66 \%$. No Brasil, não foram localizados estudos delineados especificamente para investigar a adesão ao seguimento nutricional pós-cirurgia bariátrica, questão importante para os serviços dirigidos aos obesos graves que se submetem a esse tipo de cirurgia. Essa lacuna no corpo de conhecimento científico nacional motivou o presente trabalho, que teve como objetivos estimar a prevalência de adesão ao seguimento nutricional ambulatorial pós-operatório e analisar a associação entre adesão e fatores selecionados, incluindo aspectos sociodemográficos, antropométricos e clínico-cirúrgicos.

\section{M É T O D OS}

Trata-se de um estudo observacional do tipo coorte retrospectiva. Foram elegíveis para o estudo os indivíduos obesos moderados com comorbidades e obesos mórbidos de ambos os sexos, com idade entre 19 e 70 anos, residentes no Estado de São Paulo, submetidos pelo SUS à cirurgia de DGYR por laparotomia na Divisão de Clínica Cirúrgica Il do Instituto Central do Hospital das Clínicas da Faculdade de Medicina da Universidade de São Paulo (HC-FMUSP), entre $1^{\circ}$ de janeiro de 2006 e 31 de dezembro de 2008, e matriculados no Ambulatório de Nutrição. A pesquisa foi aprovada pelo Comitê de Ética em Pesquisa (protocolo $n^{\circ}$ 194/2010) do HC-FMUSP. Os critérios de exclusão incluíram óbito e gestação nos 12 primeiros meses pós-operatórios.

Foram coletadas informações dos prontuários médicos e das fichas de evolução dietoterápica, e registradas em formulário especialmente elaborado para o estudo. As informações incluíram dados sociodemográficos (sexo, idade, endereço residencial, escolaridade, estado civil e atividade laboral); antropométricos (estatura e peso); e clínico-cirúrgicos (presença de comorbidades, estratégias para perda de peso no período pré-operatório, data da cirurgia bariátrica, data da alta hospitalar após a cirurgia e datas de comparecimento às consultas nutricionais realizadas nos 12 meses anteriores e posteriores à data da cirurgia).

Para avaliação da adesão ao seguimento nutricional ambulatorial pós-operatório (variável dependente), considerou-se a assiduidade às consultas nutricionais. Os sujeitos do estudo foram classificados em aderentes - os que compareceram a quatro ou mais consultas nos primeiros 12 meses após a data da alta hospitalar pós-operatória - ou não aderentes - os indivíduos que compareceram a três ou menos consultas no período. Essa classificação foi adotada considerando o protocolo de atendimento nutricional utilizado no serviço, que prevê seis consultas no primeiro ano pós-operatório, nas quais é realizado o moni- 
toramento clínico-nutricional incluindo medidas do peso.

As variáveis independentes de estudo foram: sexo, idade, estado conjugal (classificado de acordo com o estado civil: solteiros, viúvos, desquitados e divorciados compuseram a categoria "sem companheiro"; casados e amasiados, a categoria "com companheiro"), escolaridade, situação empregatícia ("sem remuneração" para estudantes, desempregados e donas de casa, e "com remuneração" para os que reportaram alguma atividade remunerada), distância entre a residência e o hospital, estratégias para perda de peso adotadas nos 12 meses anteriores à data da cirurgia (a variável foi categorizada em "sem intervenção", "intervenção nutricional ambulatorial exclusiva", "outras estratégias", considerando-se as outras três estratégias adotadas, isto é, internação prolongada em hospital de retaguarda, internação no período pré-cirúrgico para perda de peso e uso de balão intragástrico, associadas ou não à intervenção nutricional ambulatorial), Índice de Massa Corporal (IMC) no período pré-operatório imediato (obtido pela razão entre o peso aferido na véspera da cirurgia e a altura elevada ao quadrado e dicotomizada em " $<40 \mathrm{~kg} / \mathrm{m}^{2}$

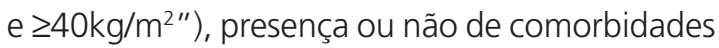
(foram consideradas todas as morbidades registradas em prontuário) e duração da internação pós-cirurgia (medida pelo período de tempo de internação da data da cirurgia até a data da alta hospitalar).

Os dados foram tabulados em planilha eletrônica de Excel e, após conferência e correção de inconsistências, foram analisados no programa estatístico STATA versão 8.0. As variáveis de estudo foram apresentadas em forma tabular e descritas por frequência absoluta e frequência relativa. Para variáveis quantitativas com distribuição normal foram calculados a média e o desvio-padrão, e a mediana para aquelas sem distribuição normal.

A prevalência de adesão para toda a população do estudo foi calculada com intervalo de 95\% de confiança (IC95\%). Em seguida, foram calculadas Razões de Prevalência (RP) com respectivos IC95\% como medida de associação entre a variável de desfecho adesão ao seguimento nutricional no período pós-operatório e cada variável independente (análise univariada). Para as variáveis ordenáveis, a significância estatística foi analisada pelo teste $\chi^{2}$ de tendência linear; para as variáveis qualitativas, a significância estatística foi analisada pelo teste $\chi^{2}$ de Pearson.

As associações entre adesão e as variáveis de interesse (análise multivariada) foram investigadas pela regressão múltipla de Poisson. Foram selecionadas as variáveis que apresentaram valor de $p \leq 0,20$ na análise univariada, além de sexo e idade. Mantidas as variáveis sexo e idade, no modelo final adotou-se um nível de significância com valor de $p \leq 0,05$.

\section{RESULTADOS}

Duzentos e quarenta e cinco indivíduos foram submetidos à DGYR por laparotomia pelo SUS na Divisão de Clínica Cirúrgica II do HCFMUSP no período de três anos, entre 2006 e 2008. Quatro sujeitos foram excluídos do estudo, três por óbito e uma mulher por gravidez nos 12 primeiros meses após a cirurgia. A população final de estudo foi composta por 241 indivíduos (Figura 1).

Os participantes eram na maioria mulheres $(80,9 \%)$, tinham média de idade de 44,4 anos $(\mathrm{DP}=11,6)$ e predomínio de baixa escolaridade $(41,9 \%)$. Pouco mais da metade dos indivíduos referiu estado conjugal "com companheiro" (51,5\%) e situação empregatícia sem remuneração (50,9\% - dados ignorados para 11 sujeitos). Aproximadamente $90 \%$ dos participantes residiam a uma distância de meio a $30,9 \mathrm{~km}$ do hospital (mediana da distância de 16,8km - dados ignorados para quatro sujeitos) (Tabela 1).

A maioria dos sujeitos da pesquisa apresentava uma ou mais comorbidades $(78,4 \%)$, sendo a hipertensão arterial sistêmica, o diabetes Mellitus tipo 2 e a colecistite crônica as mais 


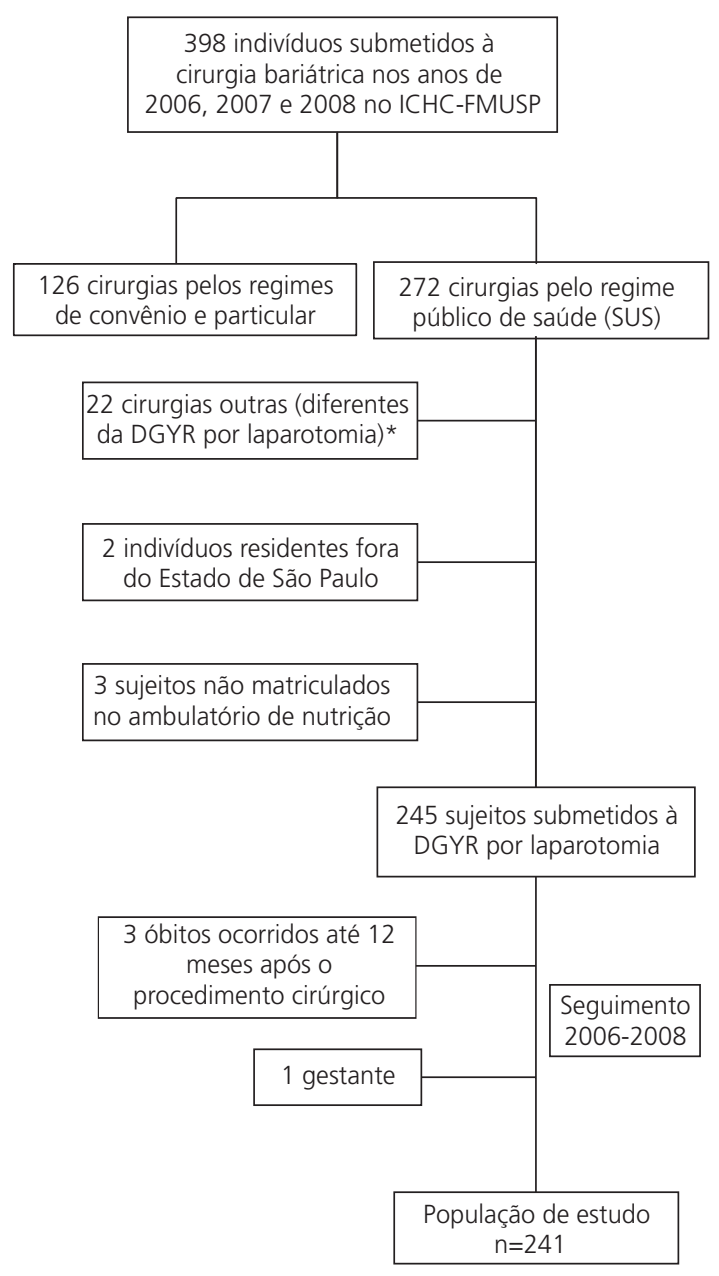

Figura 1. Universo dos indivíduos submetidos à cirurgia bariátrica no período de 1/1/2006 a 31/12/2008 e composição da população de estudo. São Paulo (SP), 2011.

*Outras cirurgias: Banda gástrica (6 indivíduos) Gastrectomia vertical (12 indivíduos) DGYR por laparoscopia (4 indivíduos)

Nota: ICHC-FMUSP: Instituto Central Hospital das Clínicas, Faculdade de Medicina da Universidade de São Paulo.

frequentes, seguidas por dislipidemias, hipotireoidismo e apneia do sono. Cerca de $60,0 \%$ dos participantes foram submetidos a uma ou mais estratégias pré-operatórias de perda de peso (dados ignorados para nove sujeitos). A média de peso da população de estudo no pré-operatório imediato à cirurgia foi de $123,1 \mathrm{~kg}(\mathrm{DP}=21,2)$ : peso mínimo de $77,8 \mathrm{~kg}$ e máximo de $214,5 \mathrm{~kg}$. O IMC pré-operatório variou entre $35,4 \mathrm{~kg} / \mathrm{m}^{2}$ e $73,7 \mathrm{~kg} /$ $\mathrm{m}^{2}$, com média de $47,2 \mathrm{~kg} / \mathrm{m}^{2}(\mathrm{DP}=6,2) ; 70$ indivíduos (29,0\%) apresentaram IMC pré-operatório
Tabela 1. Características sociodemográficas, antropométricas e clínico-cirúrgicas da população de estudo $(n=241)$. São Paulo (SP), 2011

\begin{tabular}{|c|c|c|}
\hline Variáveis & $\mathrm{n}$ & $\%$ \\
\hline \multicolumn{3}{|l|}{ Sexo } \\
\hline Feminino & 195 & 80,9 \\
\hline Masculino & 46 & 19,1 \\
\hline \multicolumn{3}{|l|}{ Faixa etária (anos) } \\
\hline $19-35$ & 67 & 27,8 \\
\hline $36-52$ & 108 & 44,8 \\
\hline $53-70$ & 66 & 27,4 \\
\hline \multicolumn{3}{|l|}{ Estado conjugal } \\
\hline Sem companheiro & 117 & 48,5 \\
\hline Com companheiro & 124 & 51,5 \\
\hline \multicolumn{3}{|l|}{ Escolaridade } \\
\hline Analfabeto e fundamental incompleto & 101 & 41,9 \\
\hline Fundamental completo e médio incompleto & 51 & 21,2 \\
\hline Ensino médio completo e superior & 89 & 36,9 \\
\hline \multicolumn{3}{|l|}{ Situação empregatícia } \\
\hline Com remuneração & 113 & 49,1 \\
\hline Sem remuneração & 117 & 50,9 \\
\hline \multicolumn{3}{|l|}{ Distância hospital-residência (km) } \\
\hline $0,5-15,9$ & 106 & 44,7 \\
\hline $16,0-30,9$ & 99 & 41,8 \\
\hline $31,0-237$ & 32 & 13,5 \\
\hline \multicolumn{3}{|l|}{ Estratégia de perda de peso pré-operatória } \\
\hline Sem intervenção & 95 & 40,9 \\
\hline Intervenção nutricional exclusiva & 45 & 19,4 \\
\hline Outras estratégias & 92 & 39,7 \\
\hline \multicolumn{3}{|l|}{ Índice de massa corporal pré-operatório $\left(\mathrm{kg} / \mathrm{m}^{2}\right)$} \\
\hline$<40$ & 26 & 10,8 \\
\hline$\geq 40$ & 215 & 89,2 \\
\hline \multicolumn{3}{|l|}{ Presença de comorbidades } \\
\hline Não & 52 & 21,6 \\
\hline $\operatorname{sim}$ & 189 & 78,4 \\
\hline \multicolumn{3}{|l|}{ Duração da internação pós-operatória (dias) } \\
\hline $2-5$ & 213 & 88,4 \\
\hline $6-141$ & 28 & 11,6 \\
\hline
\end{tabular}

maior ou igual a $50 \mathrm{~kg} / \mathrm{m}^{2}$. A maioria $(88,4 \%)$ da população de estudo apresentou internação pós-operatória de dois a cinco dias.

Com base nos registros de comparecimento às consultas nutricionais ambulatoriais nos 12 primeiros meses após a alta hospitalar pós-operatória, 135 pessoas foram classificadas como aderentes: prevalência de adesão de 56\% (IC95\% 49,7-62,3). 
502 | VM SCABIM et al.

Tabela 2. Razões de prevalência para adesão ao seguimento nutricional pós-cirurgia bariátrica, segundo características da população de estudo. São Paulo (SP), 2011.

\begin{tabular}{|c|c|c|c|c|c|}
\hline & Total & Aderente (\%) & $\mathrm{RP}$ & IC95\% & $p$ \\
\hline Sexo & & & & & 0,246 \\
\hline Feminino & 195 & 57,9 & 1 & & \\
\hline Masculino & 46 & 47,8 & 0,83 & $0,60-1,14$ & \\
\hline Faixa etária (anos) & & & & & $0,070^{*}$ \\
\hline $19-35$ & 67 & 44,8 & 1 & & \\
\hline $36-52$ & 108 & 57,4 & 1,28 & $0,94-1,75$ & \\
\hline $53-70$ & 66 & 65,2 & 1,46 & $1,06-2,00$ & \\
\hline Estado conjugal & & & & & 0,027 \\
\hline Sem companheiro & 117 & 42,2 & 1 & & \\
\hline Com companheiro & 124 & 57,8 & 1,29 & $1,03-1,63$ & \\
\hline Escolaridade & & & & & $0,793^{*}$ \\
\hline Fundamental incompleto e analfabeto & 101 & 58,4 & 1 & & \\
\hline Fundamental completo e médio incompleto & 51 & 52,9 & 0,91 & $0,67-1,23$ & \\
\hline Médio completo e superior & 89 & 55,1 & 0,94 & $0,73-1,21$ & \\
\hline Situação empregatícia & & & & & 0,371 \\
\hline Sem remuneração & 117 & 59,0 & 1 & & \\
\hline Com remuneração & 113 & 53,1 & 0,90 & $0,72-1,13$ & \\
\hline Distância hospital-residência ( $k m$ ) & & & & & $0,224^{*}$ \\
\hline $0,5-15,9$ & 106 & 50,0 & 1 & & \\
\hline $16-30,9$ & 99 & 57,6 & 1,15 & $0,89-1,48$ & \\
\hline $31-237$ & 32 & 65,6 & 1,31 & $0,96-1,80$ & \\
\hline Estratégia de perda de peso pré-operatória & & & & & 0,302 \\
\hline Sem intervenção & 95 & 51,6 & 1 & & \\
\hline Intervenção nutricional exclusiva & 45 & 64,4 & 1,25 & $0,93-1,67$ & \\
\hline Outras estratégias & 92 & 54,3 & 1,05 & $0,80-1,38$ & \\
\hline Índice de massa corporal pré-operatório $\left(\mathrm{kg} / \mathrm{m}^{2}\right)$ & & & & & 0,853 \\
\hline$<40$ & 26 & 57,7 & 1 & & \\
\hline$\geq 40$ & 215 & 55,8 & 0,97 & $0,68-1,37$ & \\
\hline Presença de comorbidades & & & & & 0,223 \\
\hline Não & 52 & 48,1 & 1 & & \\
\hline Sim & 189 & 58,2 & 1,21 & $0,89-1,65$ & \\
\hline Duração da internação pós-operatória (dias) & & & & & 0,001 \\
\hline $2-5$ & 213 & 53,1 & 1 & & \\
\hline $6-141$ & 28 & 79,6 & 1,48 & $1,18-1,87$ & \\
\hline
\end{tabular}

*Teste $\chi^{2}$ de tendência linear; RP: Razão de Procedências.

Dentre as dez variáveis independentes investigadas na análise univariada, apenas idade, duração da internação e estado conjugal apresentaram valor de $p \leq 0,20$ (Tabela 2 ). Essas três variáveis e a variável sexo foram selecionadas para a análise multivariada. Nesse modelo final, apenas duração da internação pós-operatória mostrou-se independentemente associada à adesão $(p \leq 0,05)$. Nos resultados do modelo final da aná- lise multivariada, após ajuste por idade e sexo, a duração da internação pós-operatória prolongada manteve-se associada à adesão, com razão de prevalência de 1,46 (IC95\% 1,15-1,86) (Tabela 3).

\section{I S C U S S Ã O}

A crescente incidência da obesidade nas sociedades urbanas tem sido acompanhada de 
Tabela 3. Razões de prevalência ajustadas para adesão ao seguimento nutricional pós-cirurgia bariátrica, segundo sexo, idade e duração da internação. São Paulo (SP), 2011.

\begin{tabular}{lccc}
\hline & RP & IC95\% & $p$ \\
\hline Sexo & 1 & & 0,170 \\
Feminino & 0,80 & $0,59-1,10$ & \\
Masculino & & & $0,099^{*}$ \\
Faixa etária (anos) & 1 & & \\
$19-35$ & 1,23 & $0,90-1,67$ & \\
36 - 52 & 1,37 & $0,99-1,88$ & \\
$53-70$ & & & 0,002 \\
Duração da internação & & & \\
pós-operatória (dias) & & & \\
2 - 5 & 1 & $1,15-1,86$ & \\
6 - 141 & 1,46 & & \\
\hline
\end{tabular}

*Teste $\chi^{2}$ de tendência linear; RP: Razão de Procedências.

um aumento do número de procedimentos cirúrgicos como uma importante opção de tratamento das formas graves dessa patologia. Essa realidade também ocorre no Brasil, principalmente nas Regiões Sul e Sudeste ${ }^{6}$. Por se tratar de uma cirurgia de grande porte e com risco de complicações no longo prazo, como as carências nutricionais, recomenda-se o seguimento clínico e nutricional contínuo dos indivíduos submetidos à cirurgia bariátrica ${ }^{7}$.

A prevalência de adesão às consultas nutricionais encontrada no presente estudo (56\%) foi baixa se se considerar o valor mínimo de 75\% de prevalência de adesão em cinco anos pós-cirurgia para instituições cirúrgicas pleitearem a certificação de centro de excelência pelo Surgical Review Corporation ${ }^{10}$. Por outro lado, a prevalência encontrada é semelhante àquela de estudos americanos $^{12,15,17}$ (54\%) que mediram a frequência às consultas considerando o período de 12 meses após a cirurgia. Prevalências de adesão menores (40\%) e maiores (66\%) à de nossa população foram relatadas em outros estudos ${ }^{11,13,14,16}$ nos EUA, porém os períodos adotados na definição de adesão variaram entre três meses e quatro anos de seguimento pós-operatório. Os diferentes métodos para medir adesão, incluindo a heterogeneidade da duração do período do seguimento pós-operatório e a diversidade dos protocolos quanto ao número de consultas pós-operatórias previstas, limitaram a comparabilidade dos resultados entre os estudos.

Dentre as dez variáveis analisadas no presente estudo, somente duração da internação pós-operatória igual ou superior a seis dias mostrou-se um fator preditor para adesão. As características sociodemográficas sexo, idade, estado conjugal, escolaridade e situação empregatícia, assim como distância da residência ao hospital, IMC no período pré-operatório imediato, submissão a estratégias pré-operatórias de perda de peso e presença de comorbidades não se mostraram significantemente associadas com adesão ao seguimento pós-cirúrgico.

Diante da falta de consenso na literatura científica sobre a associação entre as características sociodemográficas e a adesão às consultas no período pós-operatório, os resultados de estudos com portadores de doenças crônicas em geral também são utilizados nas discussões sobre essa questão. A ausência de associação entre sexo e adesão encontrada na nossa população de estudo corrobora resultados obtidos por Wheeler et al. ${ }^{16}$, o que não ocorre com relação aos resultados do estudo de Lara et al. ${ }^{13}$, que acharam prevalência de adesão significativamente maior nos homens $(91,3 \%)$ do que nas mulheres $(69,3 \%, p=0,04)$. Segundo a $\mathrm{OMS}^{18}$, a associação entre sexo e adesão não tem sido observada consistentemente nos estudos, o que aponta para o fato de que os aspectos culturais e outras características sociodemográficas das populações estudadas possam ser mais importantes do que gênero.

No presente estudo, não se encontrou associação entre idade e adesão, assim como no estudo de Lara et al. ${ }^{13}$. Em artigo de revisão que analisou estudos sobre adesão ao tratamento de doenças crônicas em geral, Reiners et al. ${ }^{19}$ concluíram que adolescentes e adultos jovens tendem a ser menos aderentes do que os indivíduos mais velhos. $\mathrm{A} \mathrm{OMS}^{18}$, por outro lado, sugere que as análises envolvendo idade devem incluir condições que possam estar associadas à idade, como tempo para recuperação pós-cirúrgica e recursos socioeconômicos. 
Indivíduos de estado conjugal "com companheiro" no momento da admissão ao serviço apresentaram maior adesão ao seguimento nutricional quando comparados aos indivíduos "sem companheiro" na análise univariada, mas essa associação perdeu significância estatística quando as variáveis sexo, idade e tempo de internação pós-operatória foram incluídas na análise. DiMatteo ${ }^{20}$, em metanálise que incluiu 122 estudos sobre adesão ao tratamento de doenças crônicas, observou que os sujeitos com companheiro foram mais aderentes aos tratamentos medicamentosos. Aspectos culturais relacionados à autoestima e à motivação familiar podem estar envolvidos nessa questão e isso pode ocorrer porque esses indivíduos recebem incentivo familiar para seguirem com os cuidados de saúde ${ }^{18-20}$.

Não foi verificada associação entre escolaridade e adesão nesse estudo, assim como no estudo conduzido por Wheeler et al. ${ }^{16}$. Esse resultado vai contra o senso comum de que baixa escolaridade está relacionada à baixa adesão pela dificuldade de os indivíduos com poucos anos de estudo entenderem e seguirem recomendações e terem menor nível socioeconômico, incluindo baixa renda e menor acesso a meios de transporte. Da mesma forma, o fator ter ou não atividade remunerada não mostrou associação com adesão na nossa população de estudo, enquanto Wheeler et al. ${ }^{16}$ observaram que indivíduos com emprego formal apresentaram maior adesão quando comparados aos indivíduos sem atividade remunerada. Nesse caso, diferenças no sistema assistencial entre os países podem explicar, pelo menos em parte, essa discordância. Nos EUA, as consultas são pagas pelos seguros de saúde dos indivíduos empregados, o que pode contribuir para a maior frequência às consultas.

Os estudos americanos que investigaram a associação entre distância da residência ao hospital e adesão reportaram que os indivíduos que moravam mais próximos ao hospital tendiam a ser mais aderentes ${ }^{13,14}$. Pelo fato de o Hospital das Clínicas ser um centro de referência hospitalar para os municípios vizinhos, seus usuários frequentemente contam com o transporte subsi- diado pelo serviço público local para o deslocamento ao hospital. Essa característica tem levado os profissionais de saúde do serviço a suspeitarem que os indivíduos que moram mais distantes, por terem a facilidade de transporte, faltam menos às consultas, porém no presente estudo não foi verificada associação entre adesão e distância entre endereço residencial e hospital. A comparação de resultados entre estudos de diferentes regiões que leva em conta a distância entre moradia e local da consulta deve ser feita de maneira cautelosa devido às particularidades de cada localidade e serviço.

No período do estudo, o requisito de perda de peso por meio de intervenções pré-operatórias não fazia parte dos protocolos; por esse motivo, mais de $40 \%$ da população estudada não contou com estratégias de perda de peso pré-operatórias. Considerando-se que essas estratégias levaram a um maior contato dos indivíduos com o serviço, a presença ou não de uma ou mais dessas estratégias no histórico dos participantes do estudo poderia, em tese, interferir na adesão ao seguimento pós-cirúrgico. Porém, não se observou associação entre ocorrência de estratégia de perda de peso e adesão. Não foram encontrados outros estudos que avaliassem especificamente esse fator, mas sim a relação entre perda de peso no período pré-operatório e sucesso cirúrgico medido pela perda de peso no pós-operatório. Livhits et al. ${ }^{21}$ conduziram uma metanálise com base em 14 estudos com o objetivo de investigar se a perda de peso no período pré-operatório estaria associada à perda de peso pós-cirurgia. Entre os estudos, sete apresentaram associação positiva, seis não mostraram associação, e um apresentou associação negativa entre as perdas de peso pré e pós-operatórias. Os autores inferiram que a perda de peso anterior ao procedimento cirúrgico pode ser um indicador de melhor motivação ou de seguimento às orientações, levando a melhores resultados do tratamento cirúrgico.

No presente estudo, não foi verificada associação entre IMC no período pré-operatório e adesão ao seguimento, diferentemente do estudo de Wheeler et al. ${ }^{16}$, em que os IMC pré-operató- 
rios altos foram associados à baixa adesão. Os autores sugeriram que, devido à presença de comorbidades, os indivíduos com maiores IMC geralmente fazem seguimento com outras especialidades médicas além da cirúrgica, e, após a cirurgia, podem dar preferência ao seguimento em outros serviços em detrimento do serviço de clínica cirúrgica. Magro et al. ${ }^{9}$ não estudaram a associação entre IMC e adesão, mas, ao investigar o insucesso cirúrgico baseado na perda de peso em população de 782 sujeitos, verificaram que as maiores taxas de insucesso ocorreram entre os indivíduos com $\mathrm{IMC} \geq 50 \mathrm{~kg} / \mathrm{m}^{2}$ aos 48 meses de seguimento. Os autores observaram que $60 \%$ dos sujeitos que apresentaram insucesso cirúrgico não realizaram seguimento nutricional pós-operatório, e sugerem que isso possa ter contribuído para uma menor perda de peso em longo prazo.

A presença de comorbidades no período pré-operatório não se associou à adesão no presente estudo. Não foram encontrados trabalhos que avaliassem associação entre presença de comorbidades no período pré-operatório e adesão pós-operatória. A melhora precoce do diabetes Mellitus tipo 2 e da hipertensão arterial sistêmica na grande maioria dos indivíduos operados, decorrente principalmente do procedimento cirúrgico em si, tem sido relatada em estudos que investigaram a evolução clínica dos indivíduos submetidos à cirurgia bariátrica22. A ocorrência ou não da mudança de estado de "com comorbidades" no período pré-operatório para "sem comorbidades" no pós-operatório deve ser considerada em futuros estudos de investigação da associação entre comorbidades e adesão.

No presente estudo, os indivíduos que permaneceram internados por seis ou mais dias após a cirurgia apresentaram adesão ao seguimento nutricional $46 \%$ maior quando comparados aos indivíduos que permaneceram internados por dois a cinco dias. Os motivos pelos quais os sujeitos permaneceram seis ou mais dias internados foram investigação de complicações imediatas e tratamento de complicações como fístulas e deiscências. A permanência hospitalar prolongada pode ter levado ao maior contato do indi- víduo com a equipe de cuidados e aumentado a percepção de gravidade da doença e preocupação com sua saúde, levando à maior assiduidade ao seguimento após a alta hospitalar. Não foram encontrados estudos que avaliassem a associação entre tempo de internação pós-operatório e adesão.

A natureza retrospectiva do desenho desse estudo deve ser considerada como uma limitação, pois alguns problemas foram identificados no tocante à revisão das fontes de informações, incluindo a falta de padronização das anotações clínico-cirúrgicas e registros incompletos nos prontuários médicos, além da não localização de documentos. Porém, os registros padronizados em impressos de evolução dietoterápica mostraram-se fontes de boa qualidade, pois eram preenchidos de forma regular e completa por nutricionistas treinados na rotina assistencial do Ambulatório de Nutrição desde 2006. Ainda por seu caráter retrospectivo, o presente estudo não incluiu contato com os sujeitos de pesquisa, o que impossibilitou a avaliação dos resultados da cirurgia em relação à evolução da perda de peso ou do IMC segundo adesão.

Futuros estudos nesta linha de pesquisa sobre adesão ao seguimento pós-operatório devem incluir aspectos concernentes à relação entre profissional de saúde e usuário e à percepção dos usuários da relevância dos cuidados contínuos da sua saúde. Outro aspecto importante a ser considerado em novos estudos é o impacto da adoção de estratégias de busca ativa dos faltosos aos serviços de seguimento pós-operatório como uma medida possivelmente eficaz para incrementar a adesão ao seguimento.

\section{A GRADECIMENTOS}

Ao Núcleo de Informação em Saúde do Hospital das Clínicas da Faculdade de Medicina da Universidade de São Paulo (HC-FMUSP) as informações eletrônicas; a Mariane Takesian, nutricionista, a colaboração na coleta de dados e a equipe multidisciplinar do serviço de cirurgia bariátrica do Instituto Central Hospital das Clínicas (ICHC). 


\section{COLABORADORES}

VM SCABIM e BH TESS contribuíram com a concepção do estudo, desenho e execução do projeto, análise e interpretação dos dados e redação do artigo. J ELUF-NETO contribuiu com a análise e interpretação dos dados e redação do artigo.

\section{REFERÊ NCIAS}

1. Bernardi F, Cichelero C, Vitolo MR. Comportamento de restrição alimentar e obesidade. Rev Nutr. 2005; 18(1):85-93. doi: 10.1590/S1415-52732005000 100008.

2. World Health Organization. Obesity: preventing and managing the global epidemic [Internet]. Geneva: WHO; 1998 [cited 2009 Mar 21]. Available from: <http://www.who.int//>.

3. Ogden $C L$, Carroll MD, Curtin LR, McDowell MA, Tabak CJ, Flegal KM. Prevalence of overweight and obesity in the United States, 1999-2004. JAMA. 2006; 295(13):1549-55. doi: 10.1001/295.13.1549.

4. Toledo e Associados. Pesquisa de Mercado e Opinião Pública. Pesquisa sobre obesidade no Brasil [Internet]. 2007 [acesso 2009 out 7]. Disponível em: <http://www.sbcb.org.br/>.

5. Garrido Júnior $A B$, Oliveira MR, Berti LV, Elias $A A$, Pareja JC, Matsuda $M$, et al. Derivações gastrojejunais. In: Garrido Júnior AB, Ferraz EM, Barroso FL, Marchesini JB, Szego T. Cirurgia da obesidade. São Paulo: Atheneu; 2006. p.155-61.

6. Santos LMP, Oliveira IV, Peters LR, Conde WL. Trends in morbid obesity and in bariatric surgeries covered by the Brazilian public health system. Obes Surg. 2010; 20(7):943-8. doi: 10.1007/s11695-008-95 70-3.

7. Conselho Federal de Medicina. Resolução n 1942. Normas seguras para o tratamento cirúrgico da obesidade mórbida, definindo indicações, procedimentos aceitos e equipe. Diário Oficial da União. 201012 fev; Seção 1.

8. Faintuch J, Matsuda M, Cruz MELF, Silva MM, Teivelis MP, Garrido Júnior AB, et al. Severe proteincaloric malnutrition after bariatric procedures. Obes Surg. 2004; 14(2):175-81. doi: 10.1381/096089 204322857528.

9. Magro DO, Geloneze B, Delfini R, Pareja BC, Callejas F, Pareja JC. Long-term weight regain after gastric Bypass: a 5-year prospective study. Obes Surg. 2008; 18(6):648-51. doi: 10.1007/s11695-00 7-9265-1.

10. Surgical Review Corporation. Bariatric Surgery Center of Excellence Program [Internet]. [cited 2009 Nov 25]. Available from: <http://www.surgical review.org>.
11. Dymek MP, Le Grange D, Neven K, Alverdy J. Quality of life and psychosocial adjustment in patients after Roux-en-Y Gastric Bypass: a brief report. Obes Surg. 2001; 11(1):32-9. doi: 10.1381/09608920132145 4088.

12. Shen R, Dugay G, Rajaram K, Cabrera I, Siegel N, Ren CJ. Impact of patient follow-up on weight loss after bariatric surgery. Obes Surg. 2004; 14(4):514-9. doi: $10.1381 / 096089204323013523$.

13. Lara MD, Baker MT, Larson CJ, Lambert PJ, Kothan SN. Travel distance, age, and sex as factors in follow-up visit compliance in the post-gastric Bypass population. Surg Obes Relat Dis. 2005; 1(1):17-21. doi: 10.1016/2004.11.001.

14. Gould JC, Beverstein G, Reinhardt S, Garren MJ. Impact of routine and long-term follow-up on weight loss after laparoscopic gastric Bypass. Surg Obes Relat Dis. 2007; 3(6):627-30. doi: 10.1016/20 07.07.005.

15. Harper J, Madan AK, Ternovits GA, Tichansky DS. What happens to patients who do not follow-up after bariatric surgery? Am Surg. 2007; 73(2):181-4.

16. Wheeler E, Prettyman A, Lenhard MJ, Tran K. Adherence to outpatient program postoperative appointments after bariatric surgery. Surg Obes Relat Dis. 2008; 4(4):515-20. doi: 10.1016/200 8.01.013.

17. Lanyon RI, Maxwell BM, Kraft AJ. Prediction of long-term outcome after gastric Bypass surgery. Obes Surg. 2009; 19(4):439-45. doi: 10.1007/116 95-008-9740-3.

18. World Health Organization. Adherence to longterm therapies: evidence for action. Geneve: WHO; 2003.

19. Reiners AAO, Azevedo RCS, Vieira MA, Arruda ALG. Produção bibliográfica sobre adesão/não-adesão de pessoas ao tratamento de saúde. Ciênc Saúde Coletiva. 2008; 13(Supl 2):2299-306. doi: 10.1590/ 1413-81232008000900034.

20. DiMatteo MR. Social support and patient adherence to medical treatment: a meta-analysis. Health Psychol. 2004; 23(2):207-18. doi: 10.1037/02 78-6133.23.2.207.

21. Livhits M, Mercato C, Yermilov I, Parikh JA, Dutson E, Mehran A, et al. Preoperative predictors of weight loss following bariatric surgery: systematic review. Obes Surg. 2012; 22(1):70-89. doi: 10.101 6/2009.08.014.

22. Buchwald $H$, Avidor $Y$, Braunwald $E$, Jensen $M D$, Pories W, Fahrbach K, et al. Bariatric surgery: a systematic review and meta-analysis. JAMA. 2004; 292(14):1724-37. doi: 10.1001/292.14.1724.

Recebido em: 18/1/2012 Versão final em: 4/5/2012 Aprovado em: 16/5/2012 